\title{
To Propose Improvement in Probability based object tracking technique for Multiple Object Tracking
}

\author{
Bindu sharma
}

\begin{abstract}
The object tracking is the technique which is used to track object from the image or from the video. The video consists of multiple frames and in each frame location of that object had been predicted. To predict location of the object technique of probability has been applied and this technique works on single object. In this paper, improvement has been proposed in probability based technique to track multiple objects from the video. The proposed technique had been implemented in MATLAB. The graphical results shows that proposed technique works well in terms of detection rate
\end{abstract}

Keywords: Object Tracking, Probability based technique, Detection rate, Multiple Objects

\section{Introduction}

Object tracking is an integral part of computer vision. The goal of object tracking is to find the trajectory of an object or multiple objects from a sequence of images. The results of object tracking i.e. trajectory of an object can be either of interest in its own right or can be used as the foundation for higher level analysis . The input to the object tracking method is a sequence of image frames taken after small intervals of time from the changing world. Object detection and object classification are preceding steps for tracking an object in sequence of images. Object detection is performed to check existence of real-world objects such as cars, bicycles, buildings in the sequence of images and to precisely locate such objects. The detected object can be classified into various categories such as humans, vehicles, birds, floating clouds, swaying tree and other moving objects. Object tracking is performed by monitoring spatial and temporal changes in sequence of images, including the presence of object, its position, size, shape, etc. Object tracking is used in several applications such as video surveillance, robot vision, traffic monitoring, Inpainting and Animation etc. Tracking multiple object is a complex task due to a large number of issues ranging from the different types of sensing setup to the complexity of the object appearance and behaviors . Object Detection is the task of identify objects of interest in a video sequence and to cluster pixels of these objects [1]. Since moving objects are typically the primary source of information, most methods focus on the detection of such objects. The commonly used methods of object detection are given below.

1. Frame differencing: In this method the presence of moving objects is determined by calculating the difference between two consecutive images. The difference between the two consecutive frames gives information about the location of the object. The calculations involved are simple and it is easy to implement.

2. Optical Flow: Optical flow method obtains the pattern of apparent motion of an object, its surfaces and edges in a visual scene caused by the relative motion between an observer such as an eye or a camera and the image scene. Optical flow method calculates the image optical flow field, and performs clustering process according to the optical flow distribution characteristics of image

3. Background subtraction: Background subtraction is also known as foreground detection. Background subtraction method extracts foreground objects in a particular scene [2]. A foreground object can be described as an object of attention which helps in reducing the amount of data to be processed as well as provide important information about the task under consideration.

\subsection{Object Classification Methods}

The extracted moving region may be different objects such as humans, vehicles, birds, floating clouds, swaying tree and other moving objects. The Various methods of object classification are as follows

- Shape-based classification: Different descriptions of shape information of motion regions such as representations of points, box and blob are available for classifying moving objects. Input features to the method is mixture of image-based and scene-based object parameters such as image blob area, apparent aspect ratio of blob bounding box and camera zoom. Classification is performed on each blob at every frame and results are kept in histogram.

- Motion-based classification: Non-rigid articulated object motion shows a periodic property, so this has been used as a strong cue for moving object classification. Optical flow is also very useful for object classification. Residual flow can be used to analyze rigidity and periodicity of moving entities [3]. It is 
expected that rigid objects would present little residual flow where as a non rigid moving object such as human being had higher average residual flow and even display a periodic component .

- Colour-based classification: colour is relatively constant under viewpoint changes and it is easy to acquire. Although colour is not always appropriate as the sole means of detecting and tracking objects, but the low computational cost makes colour a desirable feature to exploit when appropriate. To detect and track vehicles or pedestrians in real-time mostly colour histogram based technique is used. A colour histogram is created to describe the colour distribution within the sequence of images and to segment the image into background and objects.

- Texture-based classification Texture based classification counts the occurrences of gradient orientation in localized portions of an image. It is computed on a dense grid of uniformly spaced cells and uses overlapping local contrast normalization to improve accuracy.

\section{Literature Review}

Barga Deori et al. (2014) [4] presented a survey on object tracking on moving objects discussed the feature descriptors that are used in tracking to describe the appearance of objects which are being tracked as well as object detection techniques. They classified the tracking methods into three groups, and a providing a detailed description of representative methods in each group, and find out their positive and negative aspects.

Kiran .S. Khandar et al. (2014) [5] proposed an algorithm to track an object, moving with an unknown trajectory, within the camera's field of view. To achieve this Kalman Filter (KF) was used for tracking and estimation because of its simplicity, optimality, tractability and robustness. The Single Filter method was implemented. The Single Filter was able to track high speed an error of a couple pixels. By using this a series of measurements observed over time, containing noise and other inaccuracies, and produces estimates of unknown variables that tend to be more precise than those based on a single measurement alone .

Muammer Catak (2014) [6] proposed a probabilistic object tracking model based on condensation algorithm. A novel object tracking algorithm based on particle filtering associate with population balances was proposed. The developed algorithm was used to track objects in synthetic frames and natural video frames. Firstly, the efficiency of the developed method has been checked against synthetic video frames. Thereafter, single and multi objects scenarios have been examined on natural video frames. The colour histogram was used as the main feature of the object, and a probabilistic particle filter method incorporating with a novel population balance approach in imaging was proposed to track an object. Population balance equations (PBEs) were used to define phenomena in particulate processes.

Vishwadeep Uttamrao Landge (2014) [7] proposed a method to detect object based on background subtraction method. A reliable background updating model was established. A optimization threshold method was used to obtain behaviour of moving object and tracking. Motion of a moving object and tracking in a video stream was studied and detected. This method was beneficial for time efficient, and it works well for small numbers of moving objects. Target detection and process is realized on the video image. Video image data of the human body was processed, and its geometrical centre was obtained in different time intervals depending upon colour it are getting tracked.

Andres Alarcon Ramirez et al. (2013) [8] proposed a novel algorithm for automatic video object tracking based on a process of subtraction of successive frames, where the prediction of the direction of movement of the object being tracked was carried out by analyzing the changing areas generated as result of the object's motion, specifically in regions of interest defined inside the object being tracked in both the current and the next frame. This moving region was displaced in the direction of the object's motion predicted on the process of subtraction of successive frames. Finally, the location of the moving region of interest in the next frame that minimizes the proposed function of dissimilarity corresponds to the predicted location of the object being tracked in the next frame

\section{Problem Formulation}

A probabilistic approach is preferred to deterministic because probabilistic method considers uncertainties that may be added due to various reasons such as noise, imperfect observations etc. In a real world uncertainties are involved so to obtain accurate results it is better to consider all the possible outcomes. Objects can exhibit different dynamics at different scales so calculating the best possible solution is always a challenge. Though probabilistic tracking method is appealing there are some difficulties such as one need to have a global observation model with the same number of observations to deal with multi-object configurations varying in number, in order to obtain likelihoods of the same order of magnitude for configuration with different number of objects. Many good solutions for tracking of isolated objects or small number of objects having insignificant occlusion have been proposed in the past, multiple object tracking is still a challenging task with higher densities of people, mainly due to inter-person occlusion, bad observation viewpoints, small resolution images, entering or leaving of people etc. 
The object tracking system should be robust enough to solve these problems and robust to the noise inherent in the data. So an enhancement is proposed in the probabilistic object tracking method to track single and multiple objects more accurately, in lesser time with fewer computations. The proposed object tracking method needs to uniquely identify targets, model their interactions and handling the variability of the number of objects by defining more accurate outcomes to obtain better results.

\section{Research Methodology}

The proposed technique will improve accuracy of object tracking. In this technique multi object tracking firstly obtain single frame then on the same time in which they measure point from which the image will pitch after that process from using this we reconstruct overlaying image from that of us in which image will be find for which object will be drawn or object will be recognize that all process cover under image preprocessing. After that target identify using that in which we provide different color for each object and different shape of rectangle draw on each object which cover under extract shape after that finalize object from database or processing with using color feature or shape feature, when target found if yes then determine the object which is operated mean that object will running here and there so their detection will also move along with them, after that locate their position with some end effect or in which from the end they will locate one line with them having same color which is provide to them, after that we will find their total time, fault rate detection and detection failure rate

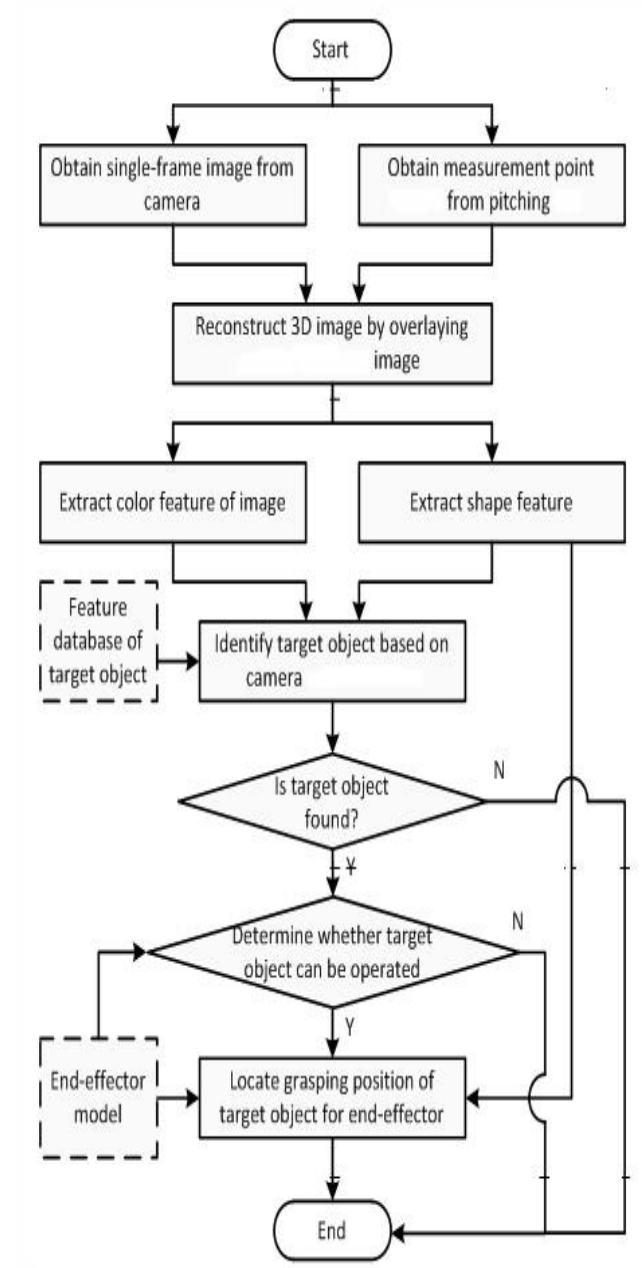

Flowchart 1: Research Methodology

\section{Implementation And Results}

In this paper, technique of multiple object tracking has been proposed. In this existing technique probability technique had been given which only work on to track single object from the video. The enhanced probability based algorithm will track multiple objects and performance of proposed algorithm is more than existing algorithm in terms of detection rate. The performance of existing and proposed algorithm has been shown graphically in figure 2 


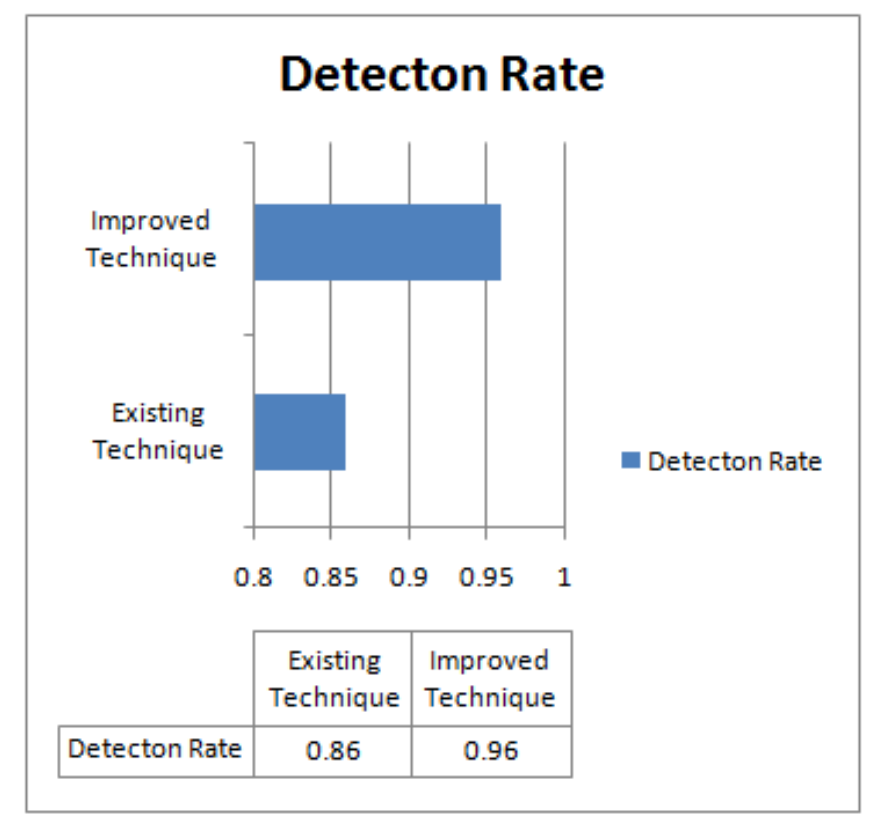

Fig 2: Comparison chart

\section{Conclusion}

In this paper, it has been concluded that existing technique will track objects from the videos on the basis of probability. In the probability based technique position of object will be predicted from the previous position of the object. This technique works well for the single object detection. To track multiple objects from the video, improvement will be proposed in the probability based technique. The graphical results show that improved technique performs batter in terms of detection rate.

\section{References}

[1] Lipton, Alan J., Hironobu Fujiyoshi, and Raju S. Patil. "Moving target classification and tracking from real-time video." Applications of Computer Vision, 1998. WACV'98. Proceedings., Fourth IEEE Workshop on. IEEE, 1998.

[2] Yilmaz, Alper, Omar Javed, and Mubarak Shah. "Object tracking: A survey."Acm computing surveys (CSUR) 38.4 (2006): 13.

[3] G. Mallikarjuna Rao and Dr.Ch.Satyanarayana, "Object Tracking System Using Approximate Median Filter, Kalman Filter and Dynamic Template Matching", I.J. Intelligent Systems and Applications, 2014

[4] Deori, Barga, and Dalton Meitei Thounaojam. "A survey on moving object tracking in video." International Journal on Information Theory (IJIT) 3.3 (2014): 31-46.

[5] Kiran .S. Khandar and Nilima R. Kharsan, "Digital Image Processing Techniques for Object Tracking System Using Kalman Filter", International Journal of Scientific Engineering and Research ISSN (Online): 2347-3878 Volume 2 Issue 6, June 2014

[6] Muammer Catak, "A probabilistic object tracking model based on condensation algorithm" Springer, 2014

[7] Vinay, D. R., and N. Lohitesh Kumar. "Object Tracking Using Background Subtraction Algorithm." International Journal of Engineering Research and General Science 3.1 (2015).

[8] Ramirez, Andres Alarcon, and Mohamed Chouikha. "A New Algorithm for Tracking Objects in Videos of Cluttered Scenes." International Journal of Information Technology (IJITMC) 1.2 (2013).

[9] A.Hema,S.Vanathi , “A Survey on Moving Object Detection Using Image Processing Techniques ”,International Journal of Innovative Research in Computer and Communication Engineering, volume. 3 Issue 7, 2015

[10] Meenatchi, K., and P. Subhashini. "Multiple Object Tracking and Segmentation in Video Sequences." International Journal 2.5 (2014).

[11] Vinay, D. R., and N. Lohitesh Kumar. "Object Tracking Using Background Subtraction Algorithm." International Journal of Engineering Research and General Science 3.1 (2015).

[12] Haritaoglu, Ismail, David Harwood, and Larry S. Davis. "W 4: Real-time surveillance of people and their activities." IEEE Transactions on pattern analysis and machine intelligence 22.8 (2000): 809-830.

[13] Singh, Tripty, S. Sanju, and Bichu Vijay. "A new algorithm designing for detection of moving objects in video." International Journal of Computer Applications 96.2 (2014).

[14] R. B. Khandoba and D.S.Patil, "Moving body/object detection and tracking”, International Journal on Information Theory (IJIT), Vol.3, No.3, July 2015. 